\title{
Nonemptiness and Compactness of Solutions Set for Nondifferentiable Multiobjective Optimization Problems
}

\author{
Xin-kun Wu, ${ }^{1}$ Jia-wei Chen, ${ }^{2}$ and Yun-zhi Zou ${ }^{1}$ \\ ${ }^{1}$ College of Mathematics, Sichuan University, Chengdu, Sichuan 610064, China \\ ${ }^{2}$ School of Mathematics and Statistics, Wuhan University, Wuhan, Hubei 430072, China \\ Correspondence should be addressed to Yun-zhi Zou, zouyz@scu.edu.cn
}

Received 13 May 2011; Accepted 10 July 2011

Academic Editor: Yongkun Li

Copyright (C) 2011 Xin-kun Wu et al. This is an open access article distributed under the Creative Commons Attribution License, which permits unrestricted use, distribution, and reproduction in any medium, provided the original work is properly cited.

A nondifferentiable multiobjective optimization problem with nonempty set constraints is considered, and the equivalence of weakly efficient solutions, the critical points for the nondifferentiable multiobjective optimization problems, and solutions for vector variational-like inequalities is established under some suitable conditions. Nonemptiness and compactness of the solutions set for the nondifferentiable multiobjective optimization problems are proved by using the FKKM theorem and a fixed-point theorem.

\section{Introduction}

The weak minimum (weakly efficient, weak Pareto) solution is an essential concept in mathematical models, economics, decision theory, optimal control, and game theory. For readers' reference, we refer to [1-11] and the references therein.

In [5], Garzón et al. studied some relationships among the weakly efficient solutions, the critical points of optimization problems, and the solutions of vector variational-like inequalities with differentiable functions. In [12], Mishra and Wang extended the work of Garzón et al. [5] to nonsmooth case. In [9], Lee et al. investigated the existence of solutions of vector optimization problems with differentiable functions. In [7], Kazmi considered the relationship between the weakly efficient solutions of a vector optimization problem and the solutions of a vector variational-like inequality with preinvex and Frechet differentiable functions. For more related work in this interesting area, we refer to $[4,10]$.

Motivated and inspired by the works mentioned above, we consider nondifferentiable multiobjective optimization problems (MOPs) with nonempty set constraints. The relationship among weakly efficient solutions, critical points of (MOP), and solutions of the vector 
variational-like inequalities (for short, (VVLI)) is presented under subinvexity, strictly pseudosubinvexity, and pseudosubinvexity conditions. By using the FKKM theorem and a fixedpoint theorem, we prove the nonemptiness and compactness of solutions set for (MOP). The results presented in this paper extend the corresponding results of $[5,7,9,12,13]$.

\section{Preliminaries}

Throughout this paper, without other specifications, let $R^{n}$ be the $n$-dimensional Euclidean space, and $R_{+}^{n}=\left\{x=\left(x_{1}, \ldots, x_{n}\right): x_{l} \geq 0, l=1, \ldots, n\right\}$. Let $K$ be a nonempty convex subset of $R^{n}$, let $H$ be a subset of $K$, and let $\operatorname{int}_{K} H$ be the relative interior of $H$ to $K$. Let $f=$ $\left(f_{1}, f_{2}, \ldots, f_{n}\right): R^{n} \rightarrow R^{n}, \eta: R^{n} \times R^{n} \rightarrow R^{n}$, and let $C: K \rightarrow 2^{R^{n}}$ such that, for each $x \in K, C(x)$ is a closed convex cone, $C(x) \neq R^{n}$, int $C(x) \neq \emptyset$, and $R_{+}^{n} \subset C(x)$. The multiobjective optimization problem (for short, (MOP)) is defined as follows:

$$
\min _{x \in K} f(x)
$$

$(\mathrm{MOP})$

We first recall some definitions and lemmas which are needed in the main results of this paper.

Definition 2.1. A point $\bar{x} \in K$ is said to be a weakly efficient (weak minimum) solution of $(\mathrm{MOP})$ if $[f(K)-f(\bar{x})] \cap(-\operatorname{int} C(\bar{x}))=\emptyset$.

Definition 2.2. A real-valued function $h: R^{n} \rightarrow R$ is said to be locally Lipschitz with respect to $\eta: R^{n} \times R^{n} \rightarrow R^{n}$ if, for each $z_{0} \in R^{n}$, there exist a neighborhood $N\left(z_{0}\right)$ of $z_{0}$ and a constant $k>0$ such that

$$
|h(x)-h(y)| \leq k\|\eta(x, y)\|, \quad \forall x, y \in N\left(z_{0}\right)
$$

Remark 2.3. If $\eta(x, y)=x-y$, then the above definition reduces to that of local Lipschitz.

Definition 2.4. A set-valued function $T: R^{n} \rightarrow 2^{R^{n}}$ is said to be locally bounded at $z_{0} \in R^{n}$ if there exist a neighborhood $N\left(z_{0}\right)$ of $z_{0}$ and a constant $k>0$ such that

$$
\|w\|<k, \quad \forall z \in N\left(z_{0}\right), \quad \forall w \in T(z)
$$

Definition 2.5. Let $T: R^{n} \rightarrow 2^{R^{n}}$ be a set-valued function. The graph of $T$ is defined as

$$
\operatorname{Gragh}(T)=\left\{(x, y): x \in R^{n}, y \in T(x)\right\}
$$

The inverse $T^{-1}$ of $T$ is defined by $x \in T^{-1}(y)$ if and only if $y \in T(x)$.

Definition 2.6. Let $X$ be a nonempty subset of topological vector space $E$. A set-valued mapping $F: X \rightarrow 2^{E}$ is called a KKM mapping if, for every finite subset $\left\{x_{1}, x_{2}, \ldots, x_{n}\right\}$ of $X, \operatorname{co}\left\{x_{1}, x_{2}, \ldots, x_{n}\right\} \subset \bigcup_{i=1}^{n} F\left(x_{i}\right)$, where co denotes the convex hull.

Definition 2.7 (see [13]). A real-valued function $h: R^{n} \rightarrow R$ is said to be subinvex at $z$ with respect to $\eta: R^{n} \times R^{n} \rightarrow R^{n}$ if there exists $\zeta \in R^{n}$, such that $h(x)-h(z) \geq\langle\zeta, \eta(x, z)\rangle$, for any 
$x \in R^{n}$, where $\zeta$ is called the $\eta$-subgradient of $h$ at $z$. The $\eta$-subdifferential of $h$ at $z$, denoted $\partial^{\eta} h(z)$, is the set of all $\zeta \in R^{n}$ such that

$$
h(x)-h(z) \geq\langle\zeta, \eta(x, z)\rangle, \quad \forall x \in R^{n} .
$$

Remark 2.8. If $h$ is locally Lipschitz, then the subinvexity of $h$ with respect to $\eta$ collapses to the invexity of $h$ with respect to $\eta$ in the sense of Clarke's generalized directional derivative with respect to $\eta[1]$. If $\eta(x, y)=x-y$ for any $x, y \in K$, then the $\eta$-subdifferential $\partial^{\eta} h(z)$ reduces to the subdifferential $\partial h(z)$ in the sense of convex analysis, where $\partial h(z)=\left\{\zeta \in R^{n}: h(x) \geq\right.$ $\left.h(z)+\langle\zeta, x-z\rangle, \forall x \in R^{n}\right\}$.

Definition 2.9. A vector-valued function $g: R^{n} \rightarrow R^{n}$ is called strictly pseudosubinvex with respect to $\eta$ if, for any $x, u \in R^{n}$ and $\left(\xi_{1}, \xi_{2}, \ldots, \xi_{n}\right) \in \partial^{\eta} g(u)$,

$$
g(x)-g(u) \in-\operatorname{int} C(u) \Longrightarrow\left(\left\langle\xi_{1}, \eta(x, u)\right\rangle,\left\langle\xi_{2}, \eta(x, u)\right\rangle, \ldots,\left\langle\xi_{n}, \eta(x, u)\right\rangle\right) \in-\operatorname{int} R_{+}^{n},
$$

where $\partial^{\eta} g(u)=\left\{\left(\xi_{1}, \xi_{2}, \ldots, \xi_{n}\right) \in\left(R^{n}\right)^{n}: g_{i}(x)-g_{i}(u) \geq\left\langle\xi_{i}, \eta(x, u)\right\rangle, \forall x \in R^{n}\right\}$.

Definition 2.10. A vector-valued function $g: R^{n} \rightarrow R^{n}$ is called pseudosubinvex with respect to $\eta$ if, for any $x, u \in R^{n}$ and $\left(\xi_{1}, \xi_{2}, \ldots, \xi_{n}\right) \in \partial^{\eta} g(u)$,

$$
g(x)-g(u) \in-\operatorname{int} C(u) \Longrightarrow\left(\left\langle\xi_{1}, \eta(x, u)\right\rangle,\left\langle\xi_{2}, \eta(x, u)\right\rangle, \ldots,\left\langle\xi_{n}, \eta(x, u)\right\rangle\right) \in-\operatorname{int} C(u),
$$

where $\partial^{\eta} g(u)=\left\{\left(\xi_{1}, \xi_{2}, \ldots, \xi_{n}\right) \in\left(R^{n}\right)^{n}: g_{i}(x)-g_{i}(u) \geq\left\langle\xi_{i}, \eta(x, u)\right\rangle, \forall x \in R^{n}\right\}$.

Remark 2.11. Since $R_{+}^{n} \subset C(x)$, it is clear that

(1) if $g: R^{n} \rightarrow R^{n}$ is strictly pseudosubinvex with respect to $\eta$, then it is pseudosubinvex with respect to $\eta$;

(2) if $g_{i}: R^{n} \rightarrow R, i=1,2, \ldots, n$, are subinvex with respect to $\eta$, then $g=\left(g_{1}, g_{2}, \ldots, g_{n}\right)$ is pseudosubinvex with respect to $\eta$.

Definition 2.12. A point $y \in K$ is called a critical point of (MOP) if there exists $\lambda=\left(\lambda_{1}\right.$, $\left.\lambda_{2}, \ldots, \lambda_{n}\right) \in R_{+}^{n}$, with $\lambda_{i}>0$ for some $i \in\{1,2, \ldots, n\}$, such that

$$
\sum_{i=1}^{n} \lambda_{i} \xi_{i}=0, \quad \forall \xi=\left(\xi_{1}, \xi_{2}, \ldots, \xi_{n}\right) \in \partial^{\eta} f(y)
$$

Lemma 2.13 (see [14](FKKM theorem)). Let $Q$ be a nonempty subset of Hausdorff topological vector space $X$. Let $G: Q \rightarrow 2^{X}$ be a KKM mapping such that for any $x \in Q, G(x)$ is closed and $G\left(x_{0}\right)$ is compact for some $x_{0} \in Q$, then there exists $x^{*} \in Q$ such that $x^{*} \in G(y)$ for all $y \in Q$, that is, $\bigcap_{x \in Q} G(x) \neq \emptyset$.

Lemma 2.14 (see [13]). Let $X$ be a nonempty and convex subset of Hausdorff topological vector space $E$, and let $S, T: X \rightarrow 2^{X}$ be two set-valued maps such that, for each $x \in X, \operatorname{coS}(x) \subset$ $T(x), S(x) \neq \emptyset, X=\bigcup\left\{\operatorname{int}_{X} S^{-1}(z): z \in X\right\}$. If there exist a nonempty compact convex set $B \subset X$ and a nonempty compact set $D \subset X$ such that, for each $z \in X \backslash D$, there exists $\bar{x} \in B$, such that $z \in \operatorname{int}_{X} S^{-1}(\bar{x})$, then there exists $\bar{z} \in X$ such that $\bar{z} \in T(\bar{z})$. 
We consider the following vector variational-like inequality (for short, (VVLI)): find $\bar{x} \in K$ such that for any $x \in K$, there exists $\xi=\left(\xi_{1}, \xi_{2}, \ldots, \xi_{n}\right) \in \partial^{\eta} f(\bar{x})$ such that

$$
\left(\left\langle\xi_{1}, \eta(x, \bar{x})\right\rangle,\left\langle\xi_{2}, \eta(x, \bar{x})\right\rangle, \ldots,\left\langle\xi_{n}, \eta(x, \bar{x})\right\rangle\right) \notin-\operatorname{int} C(\bar{x}) .
$$

\section{Relationships between (MOP) and (VVLI)}

In this section, we will investigate the properties of $\eta$-subdifferential $\partial^{\eta} h$ of the function $h$ : $R^{n} \rightarrow R$, and the relationships among weakly efficient solutions, critical points of (MOP), and the solutions of (VVILP).

Theorem 3.1. Let $h: R^{n} \rightarrow R$ be subinvex with respect to $\eta$, then the following statements are true:

(i) for each $z \in R^{n}, \partial^{\eta} h(z)$ is a nonempty closed-convex subset of $R^{n}$,

(ii) if for each $x, z \in R^{n}, \eta(x, z)+\eta(z, x)=0$, then $\partial^{\eta} h$ is $\eta$-monotone, that is, for any $x_{1}, x_{2} \in R^{n}$,

$$
\left\langle\zeta-\xi, \eta\left(x_{1}, x_{2}\right)\right\rangle \geq 0, \quad \forall \zeta \in \partial^{\eta} h\left(x_{1}\right), \quad \xi \in \partial^{\eta} h\left(x_{2}\right),
$$

(iii) if $h$ is locally Lipschitz with respect to $\eta, \eta$ is continuous in the second argument, and $\eta(x, x)=0$, for any $x \in R^{n}$, then $\operatorname{Graph}\left(\partial^{\eta} h\right)$ is closed, and $\partial^{\eta} h(\cdot)$ is upper semicontinuous,

(iv) if $\eta$ is an open map, $\eta(x, x)=0$ for any $x \in R^{n}$, and $h$ is locally Lipschitz with respect to $\eta$, then $\partial^{\eta} h(\cdot)$ is locally bounded on $R^{n}$.

Proof. Assertions (i), (ii), and (iv) are shown in [13]. We only need to prove assertion (iii). Let $\left(x_{n}, y_{n}\right) \in \operatorname{Graph}\left(\partial^{\eta} h\right)$, with $x_{n} \rightarrow x_{0}$ and $y_{n} \rightarrow y_{0}$. Since

$$
\partial^{\eta} h\left(x_{j}\right) \doteq\left\{\zeta \in R^{n}: h(x)-h\left(x_{j}\right) \geq\left\langle\zeta, \eta\left(x, x_{j}\right)\right\rangle, \forall x \in R^{n}\right\}, \quad i=1,2, \ldots, n,
$$

and $h$ is locally Lipschitz with respect to $\eta$, there exist a neighborhood $N\left(x_{0}\right)$ of $x_{0}$ and a constant $k>0$ such that

$$
|h(x)-h(z)| \leq k\|\eta(x, z)\|, \quad \forall x, z \in N\left(x_{0}\right) .
$$

Then there exists $n_{0}$ such that $x_{n} \in N\left(x_{0}\right)$ for all $n>n_{0}$, and so

$$
\left|h\left(x_{0}\right)-h\left(x_{n}\right)\right| \leq k\left\|\eta\left(x_{0}, x_{n}\right)\right\| .
$$

Consequently, we have $h\left(x_{n}\right) \rightarrow h\left(x_{0}\right)$ as $n \rightarrow \infty$. It follows from

$$
h(x)-h\left(x_{n}\right) \geq\left\langle y_{n}, \eta\left(x, x_{n}\right)\right\rangle, \quad \forall x \in R^{n}
$$

that

$$
h(x)-h\left(x_{0}\right) \geq\left\langle y_{0}, \eta\left(x, x_{0}\right)\right\rangle, \quad \forall x \in R^{n},
$$


that is, $y_{0} \in \partial^{\eta} h\left(x_{0}\right)$. Hence, $\left(x_{0}, y_{0}\right) \in \operatorname{Graph}\left(\partial^{\eta} h\right)$. In view of (i), we have that $\partial^{\eta} h(\cdot)$ is upper semicontinuous. This completes the proof.

Theorem 3.2. Let $f$ be pseudosubinvex with respect to $\eta$. If $\bar{y} \in K$ is a solution of (VVLI), then $\bar{y}$ is a weakly efficient solution of (MOP).

Proof. Let $\bar{y} \in K$ be a solution of (VVLI). If $\bar{y}$ is not a weakly efficient solution of (MOP), then there exists $\bar{x} \in K$ such that

$$
f(\bar{x})-f(\bar{y}) \in-\operatorname{int} C(\bar{y})
$$

Since $f$ is pseudosubinvex with respect to $\eta$, we have

$$
\left(\left\langle\xi_{1} \eta(\bar{x}, \bar{y})\right\rangle,\left\langle\xi_{2}, \eta(\bar{x}, \bar{y})\right\rangle, \ldots,\left\langle\xi_{n}, \eta(\bar{x}, \bar{y})\right\rangle\right) \in-\operatorname{int} C(\bar{y}), \quad \forall\left(\xi_{1}, \xi_{2}, \ldots, \xi_{n}\right) \in \partial^{\eta} f(\bar{y}),
$$

which contradicts the assumption. This completes the proof.

Corollary 3.3. Let $f_{i}, i=1,2, \ldots, n$ be subinvex with respect to $\eta$. If $\bar{y} \in K$ is a solution of (VVLI), then $\bar{y}$ is a weakly efficient solution of (MOP).

Theorem 3.4. Let $\eta$ be an open map, and continuous and affine in the first argument, let $\eta(x, y)+$ $\eta(y, x)=0$, for any $x, y \in K$. Let $f_{i}, i=1,2, \ldots, n$ be subinvex and locally Lipschitz with respect to $\eta$. If $\bar{y} \in K$ is a weakly efficient solution of (MOP), then $\bar{y}$ is a solution of (VVLI).

Proof. Let $\bar{y} \in K$ be a weakly efficient solution of (MOP). If $\bar{y}$ is not a solution of (VVLI), then

$$
\bar{y} \notin\left\{y \in K: \forall x \in K, \exists \xi_{i} \in \partial^{\eta} f_{i}(y),\left(\left\langle\xi_{1}, \eta(x, y)\right\rangle, \ldots,\left\langle\xi_{1}, \eta(x, y)\right\rangle\right) \notin-\operatorname{int} C(y)\right\}
$$

We assert that

$$
\begin{aligned}
\{y & \left.\in K: \forall x \in K, \exists \xi_{i} \in \partial^{\eta} f_{i}(y),\left(\left\langle\xi_{1}, \eta(x, y)\right\rangle, \ldots,\left\langle\xi_{n}, \eta(x, y)\right\rangle\right) \notin-\operatorname{int} C(y)\right\} \\
& =\left\{y \in K: \forall x \in K, \forall \mu_{i} \in \partial^{\eta} f_{i}(x),\left(\left\langle\mu_{1}, \eta(x, y)\right\rangle, \ldots,\left\langle\mu_{n}, \eta(x, y)\right\rangle\right) \notin-\operatorname{int} C(y)\right\} .
\end{aligned}
$$

Let

$$
\left.\bar{z} \in\left\{y \in K: \forall x \in K, \exists \xi_{i} \in \partial^{\eta} f_{i}(y),\left(\left\langle\xi_{1}, \eta(x, y)\right\rangle, \ldots,\left\langle\xi_{n}, \eta(x, y)\right\rangle\right) \notin\right\}-\operatorname{int} C(y)\right\}
$$

Suppose to the contrary that

$$
\bar{z} \notin\left\{y \in K: \forall x \in K, \forall \mu_{i} \in \partial^{\eta} f_{i}(x),\left(\left\langle\mu_{1}, \eta(x, y)\right\rangle, \ldots,\left\langle\mu_{n}, \eta(x, y)\right\rangle\right) \notin-\operatorname{int} C(y)\right\},
$$

then there exist $\widehat{x} \in K$ and $\widehat{\mu}_{i} \in \partial^{\eta} f_{i}(\widehat{x})$ such that

$$
\left(\left\langle\widehat{\mu}_{1}, \eta(\widehat{x}, \bar{z})\right\rangle, \ldots,\left\langle\widehat{\mu}_{n}, \eta(\widehat{x}, \bar{z})\right\rangle\right) \in-\operatorname{int} C(\bar{z}) .
$$


Since $\partial^{\eta} f_{i}$ is $\eta$-monotone, we have

$$
\left\langle\mu_{i}-\xi_{i}, \eta(\widehat{x}, \bar{z})\right\rangle \geq 0, \quad \forall \mu_{i} \in \partial^{\eta} f_{i}(\widehat{x}), \xi_{i} \in \partial^{\eta} f_{i}(\bar{z}),
$$

that is,

$$
\left\langle\mu_{i}, \eta(\widehat{x}, \bar{z})\right\rangle-\left\langle\xi_{i}, \eta(\widehat{x}, \bar{z})\right\rangle \in R_{+}, \quad i=1,2, \ldots, n
$$

As a consequence,

$$
\left(\left\langle\widehat{\mu}_{1}, \eta(\widehat{x}, \bar{z})\right\rangle, \ldots,\left\langle\widehat{\mu}_{n}, \eta(\widehat{x}, \bar{z})\right\rangle\right)-\left(\left\langle\xi_{1}, \eta(\widehat{x}, \bar{z})\right\rangle, \ldots,\left\langle\xi_{n}, \eta(\widehat{x}, \bar{z})\right\rangle\right) \in R_{+}^{n},
$$

and it follows that

$$
\begin{aligned}
\left(\left\langle\xi_{1}, \eta(\widehat{x}, \bar{z})\right\rangle, \ldots,\left\langle\xi_{n}, \eta(\widehat{x}, \bar{z})\right\rangle\right) & \in\left(\left\langle\widehat{\mu}_{1}, \eta(\widehat{x}, \bar{z})\right\rangle, \ldots,\left\langle\widehat{\mu}_{n}, \eta(\widehat{x}, \bar{z})\right\rangle\right)-R_{+}^{n} \\
& \subset-\operatorname{int} C(\bar{z})-R_{+}^{n} \subset-\operatorname{int} C(\bar{z}),
\end{aligned}
$$

which contradicts the assumption.

On the other hand, let

$$
y^{\prime} \in\left\{y \in K: \forall x \in K, \forall \mu_{i} \in \partial^{\eta} f_{i}(x),\left(\left\langle\mu_{1}, \eta(x, y)\right\rangle, \ldots,\left\langle\mu_{n}, \eta(x, y)\right\rangle\right) \notin-\operatorname{int} C(y)\right\}
$$

and let $r_{m} \in(0,1]$ such that $r_{m} \rightarrow 0$. For any given $x \in K$, set $x_{m}=\left(1-r_{m}\right) y^{\prime}+r_{m} x$, for any $x \in K$. Then $x_{m} \rightarrow y^{\prime}$ as $m \rightarrow \infty$. Since $\eta$ is affine in the first argument, and $\eta(x, x)=0$, we have

$$
\begin{aligned}
\left(\left\langle\mu_{1}^{m}, \eta\left(x_{m}, y^{\prime}\right)\right\rangle, \ldots,\left\langle\mu_{n}^{m}, \eta\left(x_{m}, y^{\prime}\right)\right\rangle\right) & =r_{m}\left(\left\langle\mu_{1}^{m}, \eta\left(x, y^{\prime}\right)\right\rangle, \ldots,\left\langle\mu_{n}^{m}, \eta\left(x, y^{\prime}\right)\right\rangle\right) \\
& \notin-\operatorname{int} C\left(y^{\prime}\right), \quad \forall \mu_{i}^{m} \in \partial^{\eta} f_{i}\left(x_{m}\right)
\end{aligned}
$$

that is,

$$
\left(\left\langle\mu_{1}^{m}, \eta\left(x, y^{\prime}\right)\right\rangle, \ldots,\left\langle\mu_{n}^{m}, \eta\left(x, y^{\prime}\right)\right\rangle\right) \notin-\operatorname{int} C\left(y^{\prime}\right)
$$

Since $\partial^{\eta} f_{i}$ is locally bounded, there exist a neighborhood $N\left(y^{\prime}\right)$ of $y^{\prime}$, and $k>0$ such that for any $z \in N\left(y^{\prime}\right)$, and $\psi_{i} \in \partial^{\eta} f_{i}(z)$, we have

$$
\left\|\psi_{i}\right\| \leq k, \quad i=1,2, \ldots, n
$$

Then there exists $m_{0}$, such that $x_{m} \in N\left(y^{\prime}\right)$ for any $m \geq m_{0}$, and so

$$
\left\|\mu_{i}^{m}\right\| \leq k, \quad i=1,2, \ldots, n .
$$


Consequently, $\left\{\mu_{i}^{m}\right\}$ has a convergent subsequence. Without loss of generality, let $\mu_{i}^{m} \rightarrow \mu_{i}^{\prime}$. By Theorem 3.1 (iii), we have $\mu_{i}^{\prime} \in \partial^{\eta} f_{i}\left(y^{\prime}\right)$. Since $R^{n} \backslash \operatorname{int} C\left(y^{\prime}\right)$ is closed, it follows that

$$
y^{\prime} \in\left\{y \in K: \forall x \in K, \exists \xi_{i} \in \partial^{\eta} f_{i}(y),\left(\left\langle\xi_{1}, \eta(x, y)\right\rangle, \ldots,\left\langle\xi_{n}, \eta(x, y)\right\rangle\right) \notin-\operatorname{int} C(y)\right\} .
$$

Thus, it follows from (3.9) that

$$
\bar{y} \notin\left\{y \in K: \forall x \in K, \forall \mu_{i} \in \partial^{\eta} f_{i}(x),\left(\left\langle\mu_{1}, \eta(x, y)\right\rangle, \ldots,\left\langle\mu_{n}, \eta(x, y)\right\rangle\right) \notin-\operatorname{int} C(y)\right\}
$$

that is, there exist $\bar{x} \in K$ and $\bar{\zeta}_{i} \in \partial^{\eta} f_{i}(\bar{x})$ such that

$$
\left(\left\langle\bar{\zeta}_{1}, \eta(\bar{x}, \bar{y})\right\rangle, \ldots,\left\langle\bar{\zeta}_{n}, \eta(\bar{x}, \bar{y})\right\rangle\right) \in-\operatorname{int} C(\bar{y})
$$

Since

$$
f_{i}(\bar{y})-f_{i}(\bar{x}) \geq\left\langle\bar{\zeta}_{i}, \eta(\bar{y}, \bar{x})\right\rangle, \quad \eta(\bar{y}, \bar{x})+\eta(\bar{x}, \bar{y})=0,
$$

we have

$$
\left(f_{1}(\bar{x})-f_{1}(\bar{y}), \ldots, f_{n}(\bar{x})-f_{n}(\bar{y})\right)-\left(\left\langle\bar{\zeta}_{1}, \eta(\bar{x}, \bar{y})\right\rangle, \ldots,\left\langle\bar{\zeta}_{n}, \eta(\bar{x}, \bar{y})\right\rangle\right) \in-R_{+}^{n} .
$$

Thus,

$$
f(\bar{x})-f(\bar{y}) \in-R_{+}^{n}-\operatorname{int} C(\bar{y}) \subset-\operatorname{int} C(\bar{y})
$$

that is,

$$
f(\bar{x})-f(\bar{y}) \in-\operatorname{int} C(\bar{y})
$$

which contradicts the assumption. This completes the proof.

Theorem 3.5. Let $f_{i}, i=1,2, \ldots, n$ be subinvex with respect to $\eta$, and let $f$ be strictly pseudosubinvex with respect to $\eta$. If $\bar{y} \in K$ is a critical point of (MOP), then $\bar{y}$ is a weakly efficient solution of (MOP).

Proof. Let $\bar{y} \in K$ be a critical point of (MOP). If $\bar{y}$ is not the weakly efficient solution of (MOP), then there exists $\bar{x} \in K$ such that

$$
f(\bar{x})-f(\bar{y}) \in-\operatorname{int} C(\bar{y})
$$

By the strict pseudosubinvexity of $f$ with respect to $\eta$, one has

$$
\langle\xi, \eta(\bar{x}, \bar{y})\rangle \in-\operatorname{int} R_{+}^{n}, \quad \forall \xi=\left(\xi_{1}, \xi_{2}, \ldots, \xi_{n}\right) \in \partial^{\eta} f(\bar{y}),
$$


that is,

$$
\left(\left\langle\xi_{1}, \eta(\bar{x}, \bar{y})\right\rangle, \ldots,\left\langle\xi_{n}, \eta(\bar{x}, \bar{y})\right\rangle\right) \in-\operatorname{int} R_{+}^{n}
$$

Thus, we have

$$
\left\langle\xi_{i}, \eta(\bar{x}, \bar{y})\right\rangle<0, \quad i=1,2, \ldots, n \text {. }
$$

Since $\bar{y} \in K$ is a critical point of (MOP), there is $\lambda=\left(\lambda_{1}, \lambda_{2}, \ldots, \lambda_{n}\right), i \in 1,2, \ldots, n$, with $\lambda_{i}>0$ for some $i \in\{1,2, \ldots, n\}$, such that

$$
\sum_{i=1}^{n} \lambda_{i} \xi_{i}=0
$$

Set $S_{\lambda}=\sum_{i=1}^{n} \lambda_{i}>0$, then

$$
\sum_{i=1}^{n} \frac{\lambda_{i}}{S_{\curlywedge}}=1, \quad \sum_{i=1}^{n} \frac{\lambda_{i}}{S_{\curlywedge}} \xi_{i}=0
$$

and so

$$
0=\left\langle\sum_{i=1}^{n} \frac{\lambda_{i}}{S_{\lambda}} \xi_{i}, \eta(\bar{x}, \bar{y})\right\rangle<0, \quad i=1,2, \ldots, n,
$$

which is a contradiction. This completes the proof.

Remark 3.6. If for each $y \in K, C(y)=R_{+}^{n}$, then the following statements are true in the sense of Clarke's generalized directional derivative [12]:

(1) all critical points of (MOP) are weakly efficient solutions of (MOP) if and only if $f$ is strictly pseudoinvex with respect to $\eta$;

(2) if $f$ is strictly pseudoinvex with respect to $\eta$, and locally Lipschitz, then the critical points, the weakly efficient solutions of (MOP), and the solutions of (VVLI) are equivalent.

\section{Existence of Weakly Efficient Solutions for (MOP)}

In this section, we present several existence theorems for (MOP), by using the FKKM theorem and a fixed-point theorem.

Theorem 4.1. Let $K \subset R^{n}$ be nonempty convex. Suppose that the following conditions are satisfied:

(i) $\eta(x, x)=0$ for any $x \in K$,

(ii) $x \mapsto \eta(x, \cdot)$ is affine, $y \mapsto \eta(\cdot, y)$ is continuous, 
(iii) the set-valued function $W: K \rightarrow 2^{R^{n}}$ is given by $W(x)=R^{n} \backslash(-\operatorname{int} C(x))$ for any $x \in K$ such that $\operatorname{Graph}(W)$ is closed,

(iv) $f_{i}: R^{n} \rightarrow R, i=1,2, \ldots, n$ are subinvex and locally Lipschitz with respect to $\eta$,

(v) there exists a nonempty closed bounded set $D \subset K$ such that, for each $y \in K \backslash D$, there exists $\bar{x} \in D$, and for any $\zeta_{i} \in \partial^{\eta} f_{i}(y), i \in\{1,2, \ldots, n\}$, such that

$$
\left(\left\langle\zeta_{1}, \eta(\bar{x}, y)\right\rangle,\left\langle\zeta_{2}, \eta(\bar{x}, y)\right\rangle, \ldots,\left\langle\zeta_{1}, \eta(\bar{x}, y)\right\rangle\right) \in-\operatorname{int} C(y)
$$

then the solutions set of (MOP) is nonempty compact.

Proof. Define a set-valued mapping $G: K \rightarrow 2^{D}$ by

$$
\begin{gathered}
G(z)=\left\{y \in D: \exists \xi_{i} \in \partial^{\eta} f_{i}(y),\left(\left\langle\xi_{1}, \eta(z, y)\right\rangle,\left\langle\xi_{2}, \eta(z, y)\right\rangle, \ldots,\left\langle\xi_{n}, \eta(z, y)\right\rangle\right)\right. \\
\notin-\operatorname{int} C(y)\}, \quad \forall z \in K .
\end{gathered}
$$

For any finite set $\left\{y_{1}, y_{2}, \ldots, y_{n}\right\} \subset K$, let $N \doteq \operatorname{co}\left(D \cup\left\{y_{1}, y_{2}, \ldots, y_{n}\right\}\right)$, then $N \subset K$ is a compact convex set. Define another set-valued mapping $F: N \rightarrow 2^{N}$ by,

$$
\begin{gathered}
F(z)=\left\{y \in N: \exists \xi_{i} \in \partial^{\eta} f_{i}(y),\left(\left\langle\xi_{1}, \eta(z, y)\right\rangle,\left\langle\xi_{2}, \eta(z, y)\right\rangle, \ldots,\left\langle\xi_{n}, \eta(z, y)\right\rangle\right)\right. \\
\notin-\operatorname{int} C(y)\}, \quad \forall z \in N .
\end{gathered}
$$

Obviously, $z \in F(z)$ for any $z \in N$, that is, $F(z)$ is nonempty. Let $\left\{y_{\lambda}\right\}_{\lambda \in \Delta} \subset F(z)$ be a net such that

$$
y_{\lambda} \longrightarrow y_{0} \in N, \quad \xi_{i}^{\lambda} \in \partial^{\eta} f_{i}\left(y_{\curlywedge}\right)
$$

From Theorem 3.1(iii), we conclude that Graph $\left(\partial^{\eta} f_{i}\right)$ is closed. Therefore, there exists $\xi_{i}^{0} \in$ $\partial^{\eta} f_{i}\left(y_{0}\right)$, such that $\xi_{i}^{\lambda} \rightarrow \xi_{i}^{0}, i=1,2, \ldots, n$. It follows from the closedness of Graph $\left(\partial^{\eta} f_{i}\right)$ that

$$
\left(\left\langle\xi_{1}^{0}, \eta\left(z, y_{0}\right)\right\rangle,\left\langle\xi_{2}^{0}, \eta\left(z, y_{0}\right)\right\rangle, \ldots,\left\langle\xi_{n}^{0}, \eta\left(z, y_{0}\right)\right\rangle\right) \notin-\operatorname{int} C\left(y_{0}\right)
$$

Thus, $y_{0} \in F(z)$, that is, $F(z)$ is closed, and so $F(z)$ is compact, since $N$ is compact.

It is easy to prove that, for any finite set $\left\{u_{1}, u_{2}, \ldots, u_{m}\right\} \subset N, \operatorname{co}\left\{u_{1}, u_{2}, \ldots, u_{m}\right\} \subset$ $\bigcup_{i=1}^{m} F\left(u_{i}\right)$. In fact, if there exists $\lambda_{i} \geq 0, i=1,2, \ldots, m$, with $\sum_{i=1}^{m} \lambda_{i}=1$ such that

$$
\bar{u}=\sum_{i=1}^{m} \lambda_{i} u_{i} \notin \bigcup_{i=1}^{m} F\left(u_{i}\right)
$$

then for any $\zeta_{i} \in \partial^{\eta} f_{i}(\bar{u})$,

$$
\left(\left\langle\zeta_{1}, \eta\left(u_{i}, \bar{u}\right)\right\rangle,\left\langle\zeta_{2}, \eta\left(u_{i}, \bar{u}\right)\right\rangle, \ldots,\left\langle\zeta_{n}, \eta\left(u_{i}, \bar{u}\right)\right\rangle\right) \in-\operatorname{int} C(\bar{u}) .
$$


By the convexity of $C$,

$$
\sum_{i=1}^{m} \lambda_{i}\left(\left\langle\zeta_{1}, \eta\left(u_{i}, \bar{u}\right)\right\rangle,\left\langle\zeta_{2}, \eta\left(u_{i}, \bar{u}\right)\right\rangle, \ldots,\left\langle\zeta_{n}, \eta\left(u_{i}, \bar{u}\right)\right\rangle\right) \in-\operatorname{int} C(\bar{u})
$$

Since $\eta$ is affine in the first argument, we have

$$
\left(\left\langle\zeta_{1}, \eta\left(\sum_{i=1}^{m} \lambda_{i} u_{i}, \bar{u}\right)\right\rangle,\left\langle\zeta_{2}, \eta\left(\sum_{i=1}^{m} \lambda_{i} u_{i}, \bar{u}\right)\right\rangle, \ldots,\left\langle\zeta_{n}, \eta\left(\sum_{i=1}^{m} \lambda_{i} u_{i}, \bar{u}\right)\right\rangle\right) \in-\operatorname{int} C(\bar{u}) .
$$

Now from the assumption that $\eta(x, x)=0$ for any $x \in K$, we get $0 \in-\operatorname{int} C(\bar{u})$, which is a contradiction. Therefore, $F$ is a KKM mapping. By Lemma 2.13, there exists $z_{0} \in N$ such that

$$
z_{0} \in \bigcap_{z \in N} F(z)
$$

that is,

$$
\forall z \in N, \exists \xi_{i} \in \partial^{\eta} f_{i}\left(z_{0}\right),\left(\left\langle\xi_{1}, \eta\left(z, z_{0}\right)\right\rangle,\left\langle\xi_{2}, \eta\left(z, z_{0}\right)\right\rangle, \ldots,\left\langle\xi_{n}, \eta\left(z, z_{0}\right)\right\rangle\right) \notin-\operatorname{int} C\left(z_{0}\right)
$$

From assumptions, we have $z_{0} \in D$, and moreover, $z_{0} \in \bigcap_{i=1}^{n} G\left(y_{i}\right)$, that is, $\{G(y)\}_{y \in K}$ has the finite intersection property. Consequently, $\{G(y)\}_{y \in K} \neq \emptyset$, that is, there exists $\bar{y} \in K$ such that for any $x \in K$, there exists $\bar{\xi}_{i} \in \partial^{\eta} f_{i}(\bar{y}), i=1,2, \ldots, n$, such that

$$
\left(\left\langle\bar{\xi}_{1}, \eta(x, \bar{y})\right\rangle,\left\langle\bar{\xi}_{2}, \eta(x, \bar{y})\right\rangle, \ldots,\left\langle\bar{\xi}_{n}, \eta(x, \bar{y})\right\rangle\right) \notin-\operatorname{int} C(\bar{y})
$$

From Theorem 3.1, $\bar{y}$ is a weakly efficient solution of (MOP).

Denote the solutions set of (MOP) by $J$. Let $\left\{x_{m}\right\} \subset J$, such that $x_{m} \rightarrow x_{0}$, then for any $x \in K$, there exists $\xi_{i}^{m} \in \partial^{\eta} f_{i}\left(x_{m}\right), i=1,2, \ldots, n$, such that

$$
\left(\left\langle\xi_{1}^{m}, \eta\left(x, x_{m}\right)\right\rangle,\left\langle\xi_{2}^{m}, \eta\left(x, x_{m}\right)\right\rangle, \ldots,\left\langle\xi_{n}^{m}, \eta\left(x, x_{m}\right)\right\rangle\right) \notin-\operatorname{int} C\left(x_{m}\right) .
$$

From Theorem 3.1, we have that $\partial^{\eta} f_{i}(\cdot)$ is locally bounded on $R^{n}$ and $\operatorname{Graph}\left(\partial^{\eta} f_{i}\right)$ is closed. Thus, there exists $\xi_{i}^{0} \in \partial^{\eta} f_{i}\left(x_{0}\right)$ such that $\xi_{i}^{m_{l}} \rightarrow \xi_{i}^{0}$, where $\left\{\xi_{i}^{m_{l}}\right\} \subset\left\{\xi_{i}^{m}\right\}$. Since $\eta$ is continuous in the second argument and $\operatorname{Graph}(W)$ is closed, we have

$$
\left(\left\langle\xi_{1}^{0}, \eta\left(x, x_{0}\right)\right\rangle,\left\langle\xi_{2}^{0}, \eta\left(x, x_{0}\right)\right\rangle, \ldots,\left\langle\xi_{n}^{0}, \eta\left(x, x_{0}\right)\right\rangle\right) \notin-\operatorname{int} C\left(x_{0}\right)
$$

Thus, $x_{0} \in J$, that is, $J$ is closed. From assumptions, we get $J \subset D$, and so $J$ is compact. This completes the proof. 
Example 4.2. Let $n=2, K=[0,+\infty) \times[0,1], \eta(x, y)=\left(x_{1}-y_{1}, 0\right)$, and $f(x)=\left(x_{1}, x_{1}\right)$ for any $x, y \in K$, and $C(x) \equiv R_{+}^{2}$ for any $x \in K$, where $x=\left(x_{1}, x_{2}\right), y=\left(y_{1}, y_{2}\right)$. Let $D=[0,1] \times[0,1]$. For each $y \in(0,+\infty) \times[0,1]$,

$$
f_{1}(x)-f_{1}(y)=x_{1}-y_{1} \geq\left\langle\zeta_{1}, \eta(x, y)\right\rangle=\zeta_{1}^{1}\left(x_{1}-y_{1}\right), \quad \forall \zeta_{1}=\left(\zeta_{1}^{1}, \zeta_{1}^{2}\right) \in \partial^{\eta} f_{1}(y)
$$

then

$$
\left(1-\zeta_{1}^{1}\right)\left(x_{1}-y_{1}\right) \geq 0
$$

and so $\zeta_{1}^{1}=1$. Since

$$
f_{2}(x)-f_{2}(y)=x_{1}-y_{1} \geq\left\langle\zeta_{2}, \eta(x, y)\right\rangle=\zeta_{2}^{1}\left(x_{1}-y_{1}\right), \quad \forall \zeta_{2}=\left(\zeta_{2}^{1}, \zeta_{2}^{2}\right) \in \partial^{\eta} f_{2}(y)
$$

we have $\zeta_{2}^{1}=1$. Therefore,

$$
\partial^{\eta} f(y)=\left\{\zeta=\left(\zeta_{1}, \zeta_{2}\right): \zeta_{1}=(1, a), \zeta_{2}=(1, b), \forall a, b \in R\right\}
$$

Similarly, one has

$$
\partial^{\eta} f(0)=\left\{\zeta=\left(\zeta_{1}, \zeta_{2}\right): \zeta_{1}=(c, a), \zeta_{2}=(d, b), c \leq 1, d \leq 1, \forall a, b \in R\right\} .
$$

Consequently, for any $y \in K \backslash D, \bar{x} \in D, \zeta \in \partial^{\eta} f(y)$ such that

$$
\left(\left\langle\zeta_{1}, \eta(\bar{x}, y)\right\rangle,\left\langle\zeta_{2}, \eta(\bar{x}, y)\right\rangle\right)=\left(\bar{x}_{1}-y_{1}, \bar{x}_{1}-y_{1}\right) \in-\operatorname{int} C(y)
$$

Then it is easy to check that all assumptions in Theorem 4.1 hold and $J=\{(0, v): 0 \leq v \leq 1\}$.

Corollary 4.3 (see [9]). Let $f_{i}, i=1,2, \ldots, n$ be invex in the sense of Clarke's generalized directional derivative with respect to $\eta$ and locally Lipschitz. Suppose that other conditions are the same as in Theorem 4.1, then the solutions set of (MOP) is nonempty compact.

Corollary 4.4. Let $K \subset R^{n}$ be nonempty convex. Suppose that the following conditions are satisfied:

(i) $\eta(x, x)=0$ for any $x \in K$,

(ii) $x \mapsto \eta(x, \cdot)$ is affine and $y \mapsto \eta(\cdot, y)$ is continuous,

(iii) the set-valued function $W: K \rightarrow 2^{R^{n}}$ is given by $W(x)=R^{n} \backslash(-\operatorname{int} C(x))$ for any $x \in K$ such that $\operatorname{Graph}(W)$ is closed,

(iv) $f_{i}: R^{n} \rightarrow R, i=1,2, \ldots, n$ are subinvex and locally Lipschitz with respect to $\eta$,

(v) there exists $y_{0} \in K$, such that $\left\{x \in K: \exists \zeta_{i} \in \partial^{\eta} f_{i}(x), i=1,2, \ldots, n\right.$, $\left.\left(\left\langle\zeta_{1}, \eta\left(y_{0}, y\right)\right\rangle,\left\langle\zeta_{2}, \eta\left(y_{0}, y\right)\right\rangle, \ldots,\left\langle\zeta_{n}, \eta\left(y_{0}, y\right)\right\rangle\right) \notin-\operatorname{int} C(x)\right\}$ is closed and bounded, then the solutions set of (MOP) is nonempty compact. 
Proof. Let

$$
D=\left\{x \in K: \exists \zeta_{i} \in \partial^{\eta} f_{i}(x), i=1,2, \ldots, n,\left(\left\langle\zeta_{1}, \eta\left(y_{0}, x\right)\right\rangle, \ldots,\left\langle\zeta_{n}, \eta\left(y_{0}, x\right)\right\rangle\right) \notin-\operatorname{int} C(x)\right\} .
$$

Obviously, $y_{0} \in D$, and moreover, $D$ is nonempty closed bounded. Therefore, for any $y \in$ $K \backslash D$, there exists $y_{0} \in D$ such that for any $\zeta_{i} \in \partial^{\eta} f_{i}(y), i=1,2, \ldots, n$,

$$
\left(\left\langle\zeta_{1}, \eta\left(y_{0}, y\right)\right\rangle,\left\langle\zeta_{2}, \eta\left(y_{0}, y\right)\right\rangle, \ldots,\left\langle\zeta_{n}, \eta\left(y_{0}, y\right)\right\rangle\right) \in-\operatorname{int} C(y) .
$$

By Theorem 4.1, the solutions set of (MOP) is nonempty compact. This completes the proof.

Theorem 4.5. Let $K \subset R^{n}$ be a nonempty convex set. Suppose that the following conditions are satisfied:

(i) $\eta(y, x)+\eta(x, y)=0$ for any $x, y \in K$,

(ii) $x \mapsto \eta(x, \cdot)$ is affine and $y \mapsto \eta(\cdot, y)$ is continuous,

(iii) the set-valued function $W: K \rightarrow 2^{R^{n}}$ is given by $W(x)=R^{n} \backslash(-\operatorname{int} C(x))$ for any $x \in K$ such that $\operatorname{Graph}(W)$ is closed,

(iv) $f_{i}: R^{n} \rightarrow R, i=1,2, \ldots, n$ are subinvex and locally Lipschitz with respect to $\eta$,

(v) there exists $y_{0} \in K$ such that for any $x \in K$ and $\zeta_{i} \in \partial^{\eta} f_{i}(x), i=1,2, \ldots, n$, such that

$$
\lim _{\|x\| \rightarrow \infty}\left\langle\zeta_{i}, \eta\left(y_{0}, x\right)\right\rangle<0, \quad i=1,2, \ldots, n,
$$

then the solutions set of (MOP) is nonempty compact.

Proof. Since there exists $y_{0} \in K$, such that for any $\zeta_{i} \in \partial^{\eta} f_{i}(x), i=1,2, \ldots, n$, and $x \in K$ such that

$$
\lim _{\|x\| \rightarrow \infty}\left\langle\zeta_{i}, \eta\left(y_{0}, x\right)\right\rangle<0, \quad i=1,2, \ldots, n
$$

there exists $c_{i}>0, i=1,2, \ldots, n$, such that for each $x \in K$, with $\|x\|>c_{i}$, such that

$$
\left\langle\zeta_{i}, \eta\left(y_{0}, x\right)\right\rangle<0, \quad i=1,2, \ldots, n .
$$

Taking $d=\max \left\{c_{1}+\varepsilon, c_{2}+\varepsilon, \ldots, c_{n}+\varepsilon,\left\|y_{0}\right\|+\varepsilon\right\}$, where $\varepsilon>0$, and let $D=\{x \in K:\|x\| \leq d\}$. Clearly, $D$ is closed bounded. Then for any $x \in K \backslash D$, there exists $y_{0} \in D$, and for any $\zeta_{i} \in \partial^{\eta} f_{i}(x), i=1,2, \ldots, n$, such that

$$
\left(\left\langle\zeta_{1}, \eta\left(y_{0}, x\right)\right\rangle, \ldots,\left\langle\zeta_{n}, \eta\left(y_{0}, x\right)\right\rangle\right) \in-\operatorname{int} R_{+}^{n} \subset-\operatorname{int} C(x) .
$$

By Theorem 4.1, the solutions set of (MOP) is nonempty compact. This completes the proof. 
Theorem 4.6. Let $K \subset R^{n}$ be nonempty convex. Suppose that the following conditions are satisfied:

(i) $\eta(y, x)+\eta(x, y)=0$ for any $x, y \in K$,

(ii) $\eta$ is an open mapping, $x \mapsto \eta(x, \cdot)$ is affine, and $y \mapsto \eta(\cdot, y)$ is continuous,

(iii) the set-valued function $W: K \rightarrow 2^{R^{n}}$ is given by $W(x)=R^{n} \backslash(-\operatorname{int} C(x))$ for any $x \in K$ such that $\operatorname{Graph}(W)$ is closed,

(iv) $f_{i}: R^{n} \rightarrow R, i=1,2, \ldots, n$ are subinvex and locally Lipschitz with respect to $\eta$,

(v) there exist a nonempty closed bounded set $B \subset K$ and a nonempty bounded closed set $D \subset K$ such that, for each $y \in K \backslash D$, there exist $\bar{x} \in B$ and $\zeta_{i} \in \partial^{\eta} f_{i}(y), i \in\{1,2, \ldots, n\}$ such that

$$
\left(\left\langle\bar{\zeta}_{1}, \eta(\bar{x}, y)\right\rangle, \ldots,\left\langle\bar{\zeta}_{n}, \eta(\bar{x}, y)\right\rangle\right) \in-\operatorname{int} C(y)
$$

then the solutions set of (MOP) is nonempty compact.

Proof. Define set-valued mapping $G: K \rightarrow 2^{K}$ by

$$
G(x)=\left\{y \in K: \forall \zeta_{i} \in \partial^{\eta} f_{i}(x),\left(\left\langle\zeta_{1}, \eta(x, y)\right\rangle, \ldots,\left\langle\zeta_{n}, \eta(x, y)\right\rangle\right) \notin-\operatorname{int} C(y)\right\}, \quad \forall x \in K .
$$

Obviously, $x \in G(x)$ for any $x \in K$, that is, $G(x) \neq \emptyset$.

For any net $\left\{y_{\lambda}\right\}_{\lambda \in \Delta} \subset G(x)$, with $y_{\lambda} \rightarrow y_{0}$, we have, for any $\zeta_{i} \in \partial^{\eta} f_{i}(x)$,

$$
\left(\left\langle\zeta_{1}, \eta\left(x, y_{\curlywedge}\right)\right\rangle, \ldots,\left\langle\zeta_{n}, \eta\left(x, y_{\curlywedge}\right)\right\rangle\right) \notin-\operatorname{int} C\left(y_{\curlywedge}\right),
$$

that is,

$$
\left(\left\langle\zeta_{1}, \eta\left(x, y_{\lambda}\right)\right\rangle, \ldots,\left\langle\zeta_{n}, \eta\left(x, y_{\lambda}\right)\right\rangle\right) \in W\left(y_{\lambda}\right)
$$

In view of the continuity of $\eta$ with respect to the second argument and closedness of $\operatorname{Graph}(W)$, we obtain

$$
\left(\left\langle\zeta_{1}, \eta\left(x, y_{0}\right)\right\rangle, \ldots,\left\langle\zeta_{n}, \eta\left(x, y_{0}\right)\right\rangle\right) \in W\left(y_{0}\right)
$$

and so

$$
\left(\left\langle\zeta_{1}, \eta\left(x, y_{0}\right)\right\rangle, \ldots,\left\langle\zeta_{n}, \eta\left(x, y_{0}\right)\right\rangle\right) \notin-\operatorname{int} C\left(y_{0}\right)
$$

Consequently, $y_{0} \in G(x)$, that is, $G(x)$ is closed. By Theorem 3.4, we only need to prove that (VVLI) has a solution.

Suppose to the contrary that (VVLI) has no solution, then

$$
\left\{y \in K: \forall x \in K, \exists \xi_{i} \in \partial^{\eta} f_{i}(y),\left(\left\langle\xi_{1}, \eta(x, y)\right\rangle, \ldots,\left\langle\xi_{n}, \eta(x, y)\right\rangle\right) \notin-\operatorname{int} C(y)\right\}=\emptyset .
$$


From the proof of Theorem 3.4, we have

$$
\left\{y \in K: \forall x \in K, \forall \mu_{i} \in \partial^{\eta} f_{i}(x),\left(\left\langle\mu_{1}, \eta(x, y)\right\rangle, \ldots,\left\langle\mu_{n}, \eta(x, y)\right\rangle\right) \notin-\operatorname{int} C(y)\right\}=\emptyset .
$$

Therefore, for each $y \in K,\{x \in K: y \notin G(x)\} \neq \emptyset$.

Define set-valued mappings $F_{1}, F_{2}: K \rightarrow 2^{K}$ by, respectively,

$$
\begin{aligned}
& F_{1}(y)=\left\{x \in K: \exists \zeta_{i} \in \partial^{\eta} f_{i}(x),\left(\left\langle\zeta_{1}, \eta(x, y)\right\rangle, \ldots,\left\langle\zeta_{n}, \eta(x, y)\right\rangle\right) \in-\operatorname{int} C(y)\right\}, \quad \forall y \in K, \\
& F_{2}(y)=\left\{x \in K: \forall \xi_{i} \in \partial^{\eta} f_{i}(y),\left(\left\langle\xi_{1}, \eta(x, y)\right\rangle, \ldots,\left\langle\xi_{n}, \eta(x, y)\right\rangle\right) \in-\operatorname{int} C(y)\right\}, \quad \forall y \in K .
\end{aligned}
$$

Obviously, for each $y \in K, F_{1}(y) \neq \emptyset$.

For any finite set $\left\{x_{1}, x_{2}, \ldots, x_{m}\right\} \subset F_{1}(y)$, there exist $\zeta_{i}^{j} \in \partial^{\eta} f_{i}\left(x_{j}\right), j=1,2, \ldots, m, i=$ $1,2, \ldots, n$ such that

$$
\left(\left\langle\zeta_{1}^{j}, \eta\left(x_{j}, y\right)\right\rangle,\left\langle\zeta_{2}^{j}, \eta\left(x_{j}, y\right)\right\rangle, \ldots,\left\langle\zeta_{n}^{j}, \eta\left(x_{j}, y\right)\right\rangle\right) \in-\operatorname{int} C(y)
$$

By the $\eta$-monotonicity of $\partial^{\eta} f_{i}$, we have, for each $j \in\{1,2, \ldots, m\}$,

$$
\left\langle\zeta_{i}^{j}, \eta\left(x_{j}, y\right)\right\rangle-\left\langle\xi_{i}, \eta\left(x_{j}, y\right)\right\rangle \geq 0, \quad \forall \xi_{i} \in \partial^{\eta} f_{i}(y)
$$

It follows that

$$
\left(\left\langle\zeta_{1}^{j}, \eta\left(x_{j}, y\right)\right\rangle, \ldots,\left\langle\zeta_{n}^{j}, \eta\left(x_{j}, y\right)\right\rangle\right)-\left(\left\langle\xi_{1}, \eta\left(x_{j}, y\right)\right\rangle, \ldots,\left\langle\xi_{n}, \eta\left(x_{j}, y\right)\right\rangle\right) \in R_{+}^{n} \subset C(y) .
$$

Since $\eta(x, y)+\eta(y, x)=0$, for any $x, y \in K$, we get

$$
\left(\left\langle\xi_{1}, \eta\left(y, x_{j}\right)\right\rangle, \ldots,\left\langle\xi_{n}, \eta\left(y, x_{j}\right)\right\rangle\right)-\left(\left\langle\zeta_{1}^{j}, \eta\left(y, x_{j}\right)\right\rangle, \ldots,\left\langle\zeta_{n}^{j}, \eta\left(y, x_{j}\right)\right\rangle\right) \in C(y)
$$

that is,

$$
\begin{aligned}
\left(\left\langle\xi_{1}, \eta\left(y, x_{j}\right)\right\rangle, \ldots,\left\langle\xi_{n}, \eta\left(y, x_{j}\right)\right\rangle\right) & \in\left(\left\langle\zeta_{1}^{j}, \eta\left(y, x_{j}\right)\right\rangle, \ldots,\left\langle\zeta_{n}^{j}, \eta\left(y, x_{j}\right)\right\rangle\right)+C(y) \\
& \subset \operatorname{int} C(y)+C(y) \subset \operatorname{int} C(y) .
\end{aligned}
$$

Therefore, for any $\alpha_{j} \geq 0, \Sigma_{j=1}^{m} \alpha_{j}=1, \Sigma_{j=1}^{m} \alpha_{j}\left(\left\langle\xi_{1}, \eta\left(y, x_{j}\right)\right\rangle, \ldots,\left\langle\xi_{n}, \eta\left(y, x_{j}\right)\right\rangle\right) \in \operatorname{int} C(y)$, and thus

$$
\left(\left\langle\xi_{1}, \sum_{j=1}^{m} \alpha_{j} \eta\left(x_{j}, y\right)\right\rangle, \ldots,\left\langle\xi_{n}, \sum_{j=1}^{m} \alpha_{j} \eta\left(x_{j}, y\right)\right\rangle\right) \in-\operatorname{int} C(y)
$$


Since $\eta$ is affine in the first argument, it follows that

$$
\left(\left\langle\xi_{1}, \eta\left(\sum_{j=1}^{m} \alpha_{j} x_{j}, y\right)\right\rangle, \ldots,\left\langle\xi_{n}, \eta\left(\sum_{j=1}^{m} \alpha_{j} x_{j}, y\right)\right\rangle\right) \in-\operatorname{int} C(y)
$$

We have $\Sigma_{j=1}^{m} \alpha_{j} x_{j} \in F_{2}(y)$, that is, $\operatorname{co}\left(F_{1}(y)\right) \subset F_{2}(y)$ for any $y \in K$. In view of the closedness of $G(x)$, one has $F_{1}^{-1}(x)=K \backslash G(x)$ as an open set in $K$. Therefore, $\operatorname{int}_{K} F_{1}^{-1}(x)=F_{1}^{-1}(x)$. From (4.34), we have

$$
\bigcup_{x \in K}\left\{y \in K: \exists \zeta_{i} \in \partial^{\eta} f_{i}(x),\left(\left\langle\zeta_{1}, \eta(x, y)\right\rangle, \ldots,\left\langle\zeta_{n}, \eta(x, y)\right\rangle\right) \in-\operatorname{int} C(y)\right\}=K
$$

that is,

$$
\bigcup_{x \in K} F_{1}^{-1}(x)=K
$$

Furthermore,

$$
K=\bigcup_{x \in K} F_{1}^{-1}(x)=\bigcup_{x \in K} \operatorname{int}_{K} F_{1}^{-1}(x)
$$

From assumption $(v)$, one has

$$
y \in F_{1}^{-1}(\bar{x})=\operatorname{int}_{K} F_{1}^{-1}(\bar{x})
$$

By Lemma 2.14, there exists $\bar{y} \in K$ such that $\bar{y} \in F_{2}(\bar{y})$. Since $\eta(x, y)+\eta(y, x)=0$ for any $x, y \in K$, we have $\eta(\bar{y}, \bar{y})=0$. Thus,

$$
\forall \xi_{i} \in \partial^{\eta} f_{i}(\bar{y}), \quad\left(\left\langle\xi_{1}, \eta(\bar{y}, \bar{y})\right\rangle, \ldots,\left\langle\xi_{n}, \eta(\bar{y}, \bar{y})\right\rangle\right) \in-\operatorname{int} C(\bar{y}),
$$

that is, $0 \in-\operatorname{int} C(\bar{y})$, which is a contradiction. Therefore, (VVILP) has a solution. From Theorems 3.2 and 3.4, (MOP) has a weakly efficient solution.

Similarly, we can show that $J$ is compact.This completes the proof.

Remark 4.7. As previously mentioned, the results presented in this paper extend some corresponding results in $[5,7,9,12,13]$. For instance, Theorems 4.1 and 4.6 extended the corresponding results given by Kazmi [7], and Lee et al. [13], Lee et al. [9] from preinvex functions to subinvex functions. Corollaries 4.3 and 4.4 generalized the results given by Lee et al. [13] from differentiable functions to nondifferentiable functions. 


\section{Acknowledgments}

This work was supported by the National Natural Science Foundation of China (no. 60804065); academic award for excellent PhD candidates funded by Ministry of Education of China; the Fundamental Research Fund for the Central Universities (no. 201120102020004).

\section{References}

[1] Q. H. Ansari and J. C. Yao, “On nondifferentiable and nonconvex vector optimization problems," Journal of Optimization Theory and Applications, vol. 106, no. 3, pp. 475-488, 2000.

[2] G. Y. Chen, X. X. Huang, and X. Q. Yang, Vector Optimization: Set-Valued and Variational Analysis, vol. 541 of Lecture Notes in Economics and Mathematical Systems, Springer, Berlin, Germany, 2005.

[3] J. W. Chen, Y. J. Cho, J. K. Kim, and J. Li, "Multiobjective optimization problems with modified objective functions and cone constraints and applications," Journal of Global Optimization, vol. 49, no. 1, pp. 137-147, 2011.

[4] X. H. Gong and J. C. Yao, "Connectedness of the set of efficient solutions for generalized systems," Journal of Optimization Theory and Applications, vol. 138, no. 2, pp. 189-196, 2008.

[5] G. R. Garzón, R. O. Gómez, and A. R. Lizana, "Relationships between vector variational-like inequality and optimization problems," The European Journal of Operational Research, vol. 157, no. 1, pp. 113-119, 2004.

[6] M. A. Hanson, "On sufficiency of the Kuhn-Tucker conditions," Journal of Mathematical Analysis and Applications, vol. 80, no. 2, pp. 545-550, 1981.

[7] K. R. Kazmi, "Existence of solutions for vector optimization," Applied Mathematics Letters, vol. 9, no. 6, pp. 19-22, 1996.

[8] D. T. Luc, Theory of Vector Optimization, vol. 319 of Lecture Notes in Economics and Mathematical Systems, Springer, Berlin, Germany, 1989.

[9] G. M. Lee, D. S. Kim, and H. Kuk, "Existence of solutions for vector optimization problems," Journal of Mathematical Analysis and Applications, vol. 220, no. 1, pp. 90-98, 1998

[10] L. Li and J. Li, "Equivalence and existence of weak Pareto optima for multiobjective optimization problems with cone constraints," Applied Mathematics Letters, vol. 21, no. 6, pp. 599-606, 2008.

[11] X. M. Yang, X. Q. Yang, and K. L. Teo, "Some remarks on the Minty vector variational inequality," Journal of Optimization Theory and Applications, vol. 121, no. 1, pp. 193-201, 2004.

[12] S. K. Mishra and S. Y. Wang, "Vector variational-like inequalities and non-smooth vector optimization problems," Nonlinear Analysis, vol. 64, no. 9, pp. 1939-1945, 2006.

[13] G. M. Lee, B. S. Lee, and S. S. Chang, "On vector quasivariational inequalities," Journal of Mathematical Analysis and Applications, vol. 203, no. 3, pp. 626-638, 1996.

[14] K. Fan, "A generalization of Tychonoff's fixed point theorem," Mathematische Annalen, vol. 142, pp. 305-310, 1961. 


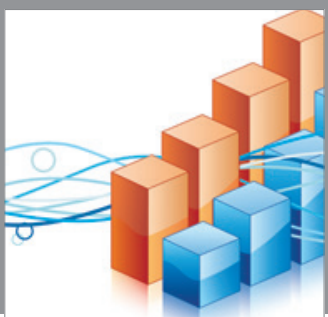

Advances in

Operations Research

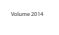

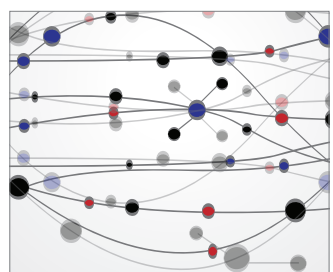

\section{The Scientific} World Journal
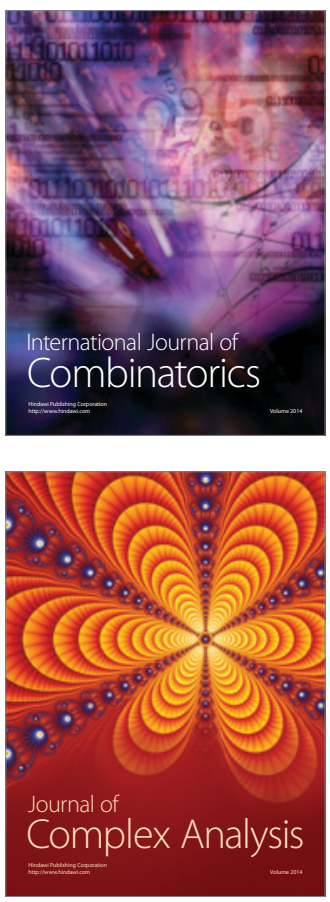

International Journal of

Mathematics and

Mathematical

Sciences
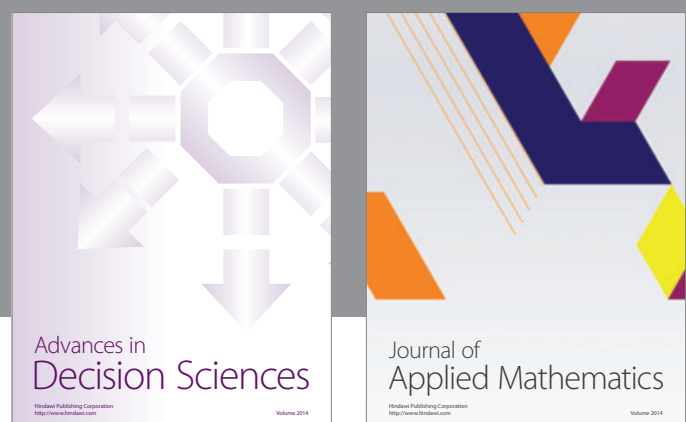

Journal of

Applied Mathematics
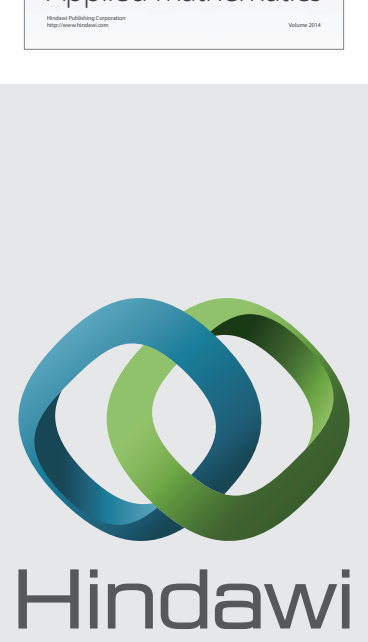

Submit your manuscripts at http://www.hindawi.com


Mathematical Problems in Engineering
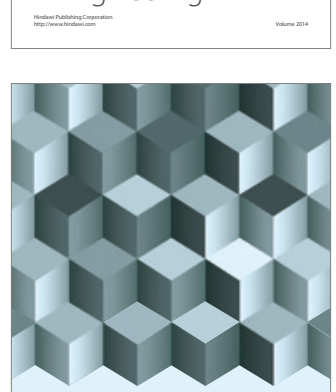

Journal of

Function Spaces
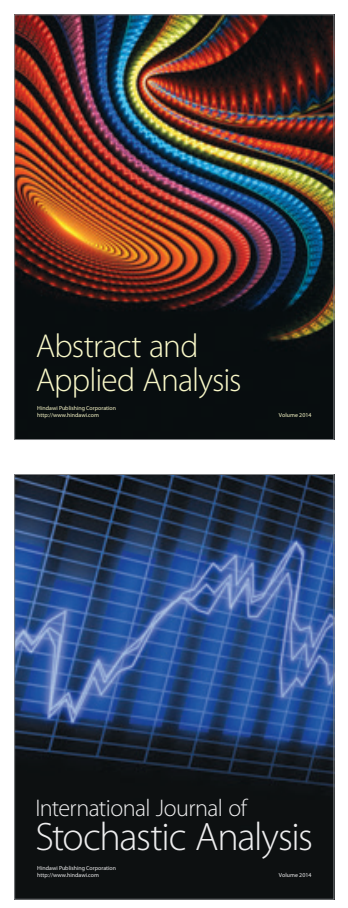

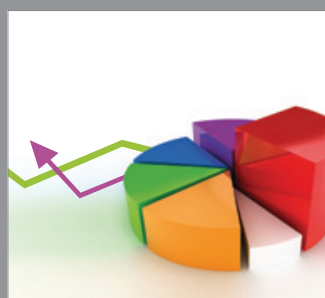

ournal of

Probability and Statistics

Promensencen
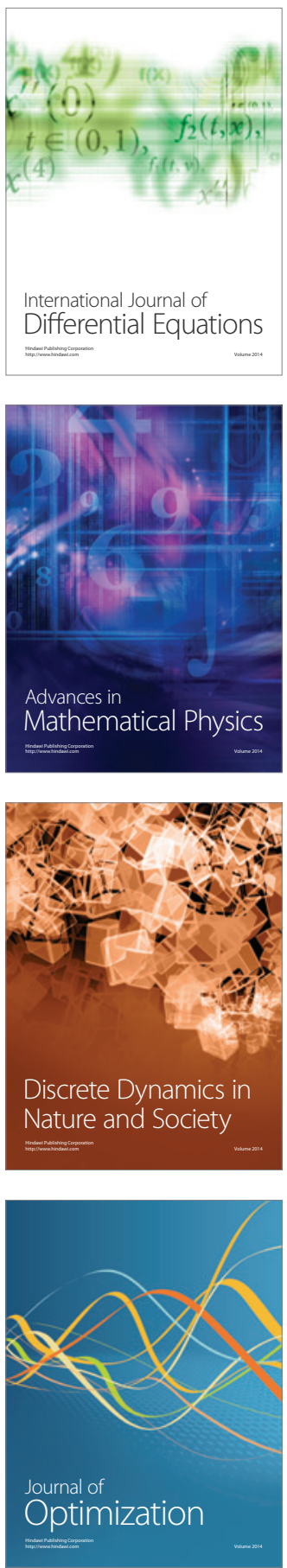